\section{J.-B. KAZMIERCZAK}

BRGM

Direction de la Recherche,

Avenue de Concyr

BP 6009 ,

45060 Orléans Cedex 02

Terrasol,

Immeuble Helios

72, avenue Pasteur

93108 Montreuil Cedex

Tél. : (33) 0149882469

E-mail:terrasol@wanados.fr

\title{
Influence sur le milieu de l'installation d'un panneau de paroi moulée en béton, dans une argile raide saturée
}

L'objet de cet article est d'apporter quelques éléments de réponse à la question suivante : la phase initiale du creusement, sous boue de bentonite, d'un panneau de paroi moulée, a-t-elle une influence sur le comportement ultérieur du sol et des structures ?

Une méthode numérique aux différences finies (modèle biphasique couplé), avec une gẻométrièe tridimensionnelle a été mise en œuvre. Les résultats qu'elle procure ont été confrontés avec des mesures de chantier. Cette analyse montre que l'approche $3 \mathrm{D}$ est acceptable pour l'évaluation des déplacements, des contraintes et des pressions interstitielles. Elle pourrait toutefois être avantageusement remplacée par une approche axisymétrique.

\section{Effect of a cast in situ concrete diaphragm wall panel on the behavior of a saturated stiff clay}

The scope of this paper is to provide several findings relating to the question : Does the initial excavation phase of a cast in situ panel using a bentonite supporting fluid have an effect on the ultimate behavior of the soil and supported structures ?

This study was performed using the Finite Difference Method and considering a three-dimensional geometry and a dual phase coupled behavior. The numerical results were compared to the measurements made on-site. The study demonstrated that a 3D approach is suitable for evaluating the displacements, stresses and pore water pressures. On the other hand, it was also show that the three-dimensional model could be replaced by a more simple axisymmetrical analysis. 


\section{Introduction}

Le problème du dimensionnement des parois moulées a été abordé de différentes manières par les concepteurs. Pourtant, qu'ils utilisent la méthode basée sur l'équilibre limite, celle aux coefficients de réaction ou encore celle du continuum élastoplastique, peu se sont intéressés à la phase initiale du creusement de la paroi. En effet, les calculs sont souvent menés en considérant que la paroi est déjà présente dans le sol alors que la réalisation de la tranchée seule modifie de façon non négligeable l'état de contrainte du massif de sol avoisinant la saignée.

Il semble aujourd'hui admis que le processus d'installation des parois moulées dans les argiles raides peut causer des déplacements significatifs dans le milieu environnant ainsi que des réductions conséquentes de la contrainte horizontale. De nombreux auteurs tels que Potts et Fourie (1984), Tedd et al. (1984), Powrie et al. (1991, 1992) et Gun et al. (1992) ont observé ces phénomènes dans les argiles raides.

Il paraît donc intéressant d'essayer de prévoir les effets de l'installation de ce type d'ouvrage dans l'argile. Aussi, des chercheurs britanniques ont mis en œuvre des études numériques pour tenter de modéliser ce processus d'installation des parois. On peut citer Higgins et al. (1989) qui modélisent l'installation de la paroi en imposant une pression hydrostatique représentant le béton sur les flancs de la tranchée. Leur analyse est du type déformation plane, bidimensionnelle. Powrie et Li (1991) ont, pour leur part, réduit le coefficient latéral de pression des terres dans le sol derrière l'écran.

Plus récemment, $\mathrm{Ng}$ et al. (1995) ont abordé le problème de l'installation d'une série de panneaux en béton dans l'argile. Leur analyse numérique, très complète, est fondée sur deux analyses simplifiées de section plane.

Le propos de cet article est d'étudier numériquement l'influence du creusement sous boue de bentonite et de la mise en place d'un panneau en béton moulé dans une argile raide saturée. Pour ce faire une simulation tridimensionnelle biphasique (méthode du continuum élastoplastique - différences finies - logiciel FLAC 3D V1.05) d'un panneau de l'une des parois moulées du métro de Lille est réalisée. Le modèle de comportement du sol est celui de Mohr-Coulomb. Les paramètres ont été calés à partir d'essais de laboratoire et in situ.

Une confrontation avec des mesures en place effectuées sur un chantier similaire à celui que nous modélisons est proposée de manière à déterminer la validité de ce type d'approche.

\section{2}

\section{Présentation du chantier de référence}

Les travaux présentés dans cet article se basent sur le chantier de la station Alma-Gare du métro de Lille. Cette station traverse des formations constituées par le matériau Argile des Flandres. Elle se situe à Roubaix dans le quartier de la gare.

\section{Géometrie et phasage du chantier}

La géométrie du projet, et le schéma d'implantation de l'instrumentation sont représentés sur la figure 1.

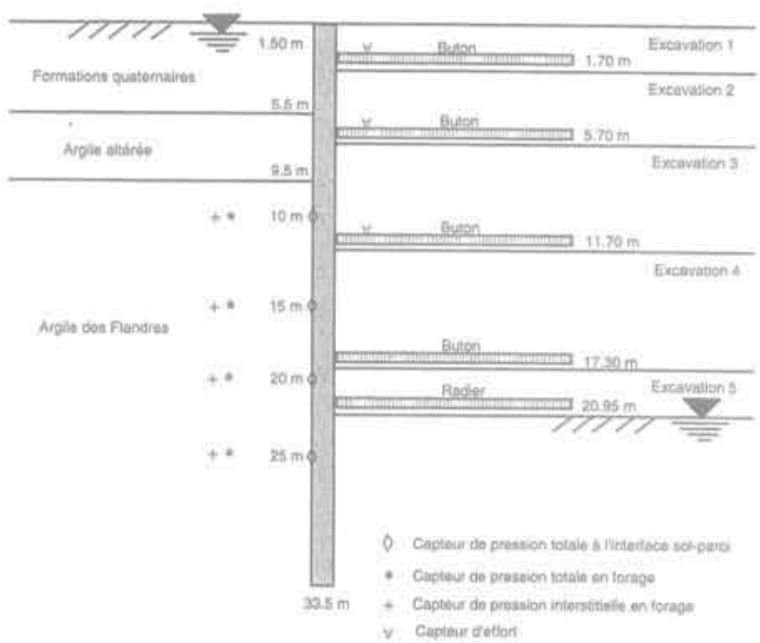

FIG.1 Coupe schématique de la station AlmaGare et instrumentation du milieu. Schematical cross section of Alma-Gare station and instrumentation of the site.

On y distingue :

- des capteurs de pression situés à l'interface sol-paroi; - des capteurs de pression en forage situés à $1,80 \mathrm{~m}$ de la paroi :

- des capteurs d'effort sur les butons.

La paroi, d'une hauteur de $33,5 \mathrm{~m}$ dans sa partie la plus profonde et de 1,22 m d'épaisseur, est inscrite dans trois couches de sol:

- de la surface à $5,50 \mathrm{~m}$ de profondeur : formations quaternaires ;

- de $5,50 \mathrm{~m}$ à $9,50 \mathrm{~m}$ de profondeur : argile altérée : - ensuite : Argile des Flandres,

La paroi est maintenue durant la phase travaux par quatre lits de butons. Un radier est réalisé en fond de fouille après la dernière excavation.

\section{2}

\section{Instrumentation}

\subsection{1.}

Les capteurs de pression

La position des différents capteurs est repérée sur la figure 1. L'instrumentation comprend douze capteurs de pression hydraulique à contre-pression pneumatique. Ils ont été placés à $10,15,20$ et $25 \mathrm{~m}$ de profondeur de la façon suivante :

- quatre capteurs de pression totale situés à l'interface sol-paroi ;

- quatre capteurs de pression interstitielle situés dans des forages à $1,80 \mathrm{~m}$ du dos de la paroi ; 
- quatre capteurs de pression totale situés dans des forages à $1,80 \mathrm{~m}$ du dos de la paroi.

Les capteurs de pression totale accolés à la paroi ont été mis en place au moment du remplissage en béton de la paroi moulée. Les huit autres capteurs ont été placés dans des forages distincts. Les capteurs de pression totale en forage ont préalablement été enrobés dans un coulis de module de compressibilité correspondant à celui du sol à la profondeur considérée.

Tous ces capteurs ont été reliés à une centrale d'acquisition de données, de marque Glötzl, programmée pour effectuer une série de mesures toutes les douze heures.

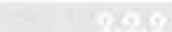

\section{Les capteurs d'efforts}

Sur chaque niveau de butons sont placées des cordes vibrantes. Elles sont disposées à $120^{\circ}$ sur deux rangées de butons distinctes. Ces capteurs fournissent donc en retour des fréquences qui sont retranscrites, à partir d'une table de conversion, en déformations et efforts.

\section{Réalisation d'une paroi moulée}

Les parois en béton, moulées dans le sol, sont réalisées par panneaux successifs de largeur variable. Dans la plupart des cas, l'opération s'effectue en trois étapes itératives :

- creusement du premier panneau primaire d'une longueur de x mètres, bétonnage du panneau primaire avec mise en place d'un joint d'étanchéité ;

- creusement du second panneau primaire d'une longueur de x mètres, bétonnage du second panneau primaire avec mise en place d'un joint d'étanchéité ;

- creusement du panneau secondaire de longueur variable, bétonnage du panneau secondaire.

Dans le cas qui nous intéresse, nous observerons l'effet du creusement sous boue de bentonite d'un panneau de $\mathrm{x}=3 \mathrm{~m}$ de longueur, de $1,22 \mathrm{~m}$ de largeur et de $33,50 \mathrm{~m}$ de hauteur.

\section{3}

\section{Modélisation numérique tridimensionnelle biphasique, du creusement d'une tranchée, sous boue de bentonite}

\section{1}

\section{Mise en œuvre de la modélisation numérique tridimensionnelle biphasique du continuum élastoplastique}

\section{$3: 1.1$}

\section{Géométrie et conditions aux limites}

Le problème étant symétrique par rapport à un axe vertical passant par le point 0 de la figure 3 , seul le

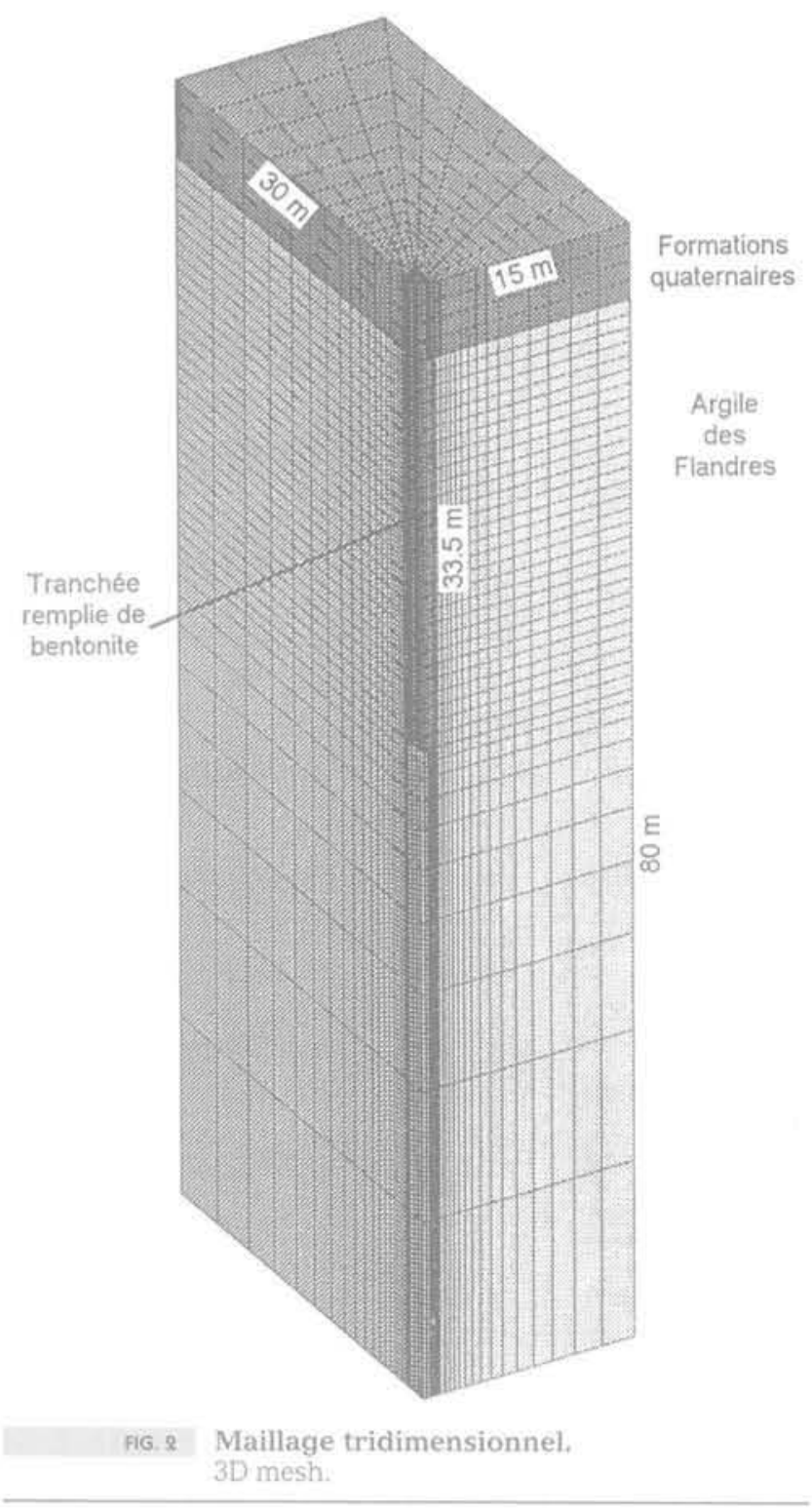

quart de la géométrie totale est modélisé, Les dimensions totales de la tranchée sont les suivantes: $1,22 \mathrm{~m} x$ $3,00 \mathrm{~m} \times 33,50 \mathrm{~m}$. La figure 2 représente une vue d'ensemble du maillage qui comprend au total 11911 nouds et 10248 éléments à 8 ncuds. L'étendue du domaine est de $15,00 \mathrm{~m} \times 30,00 \mathrm{~m} \times 80,00 \mathrm{~m}$.

La figure 3 reprend la partie supérieure du maillage. Elle permet d'en observer la finesse, ainsi que la répartition des nceuds et des zones.

Les conditions aux limites mécaniques imposées sur le massif sont les suivantes (voir Fig. 4):

- déplacements suivant z imposés nuls sur la face 4 ; - déplacements suivant $x$ imposés nuls sur les faces 5 et 6 ; - déplacements suivant y imposés nuls sur les faces 2 et 3.

Nota : Les conditions aux limites ne sont pas imposées en contraintes mais en déplacements.

Les conditions hydrauliques imposées sont : flux nul partout, y compris sur les faces de la tranchée soumises à l'effet de la bentonite.

Par ailleurs, la pression interstitielle est initialisée puis, fixée dans les formations quaternaires. 


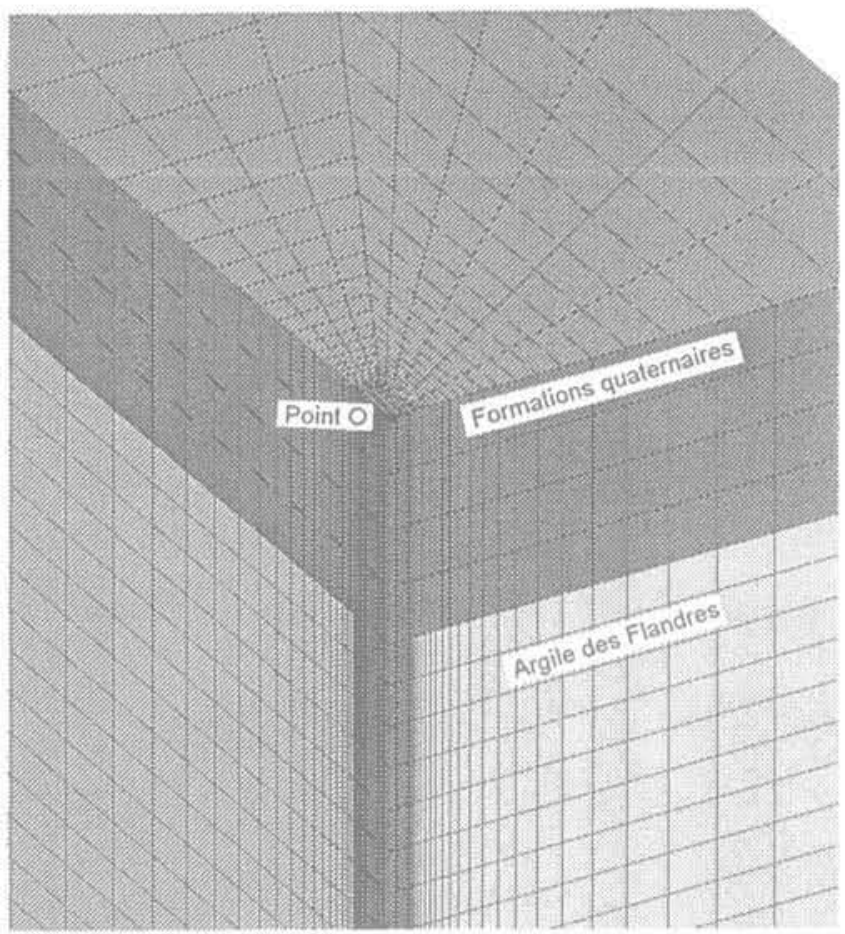

FIG.3 Partie supérieure du maillage tridimensionnel. Upper part of the 3D mesh.

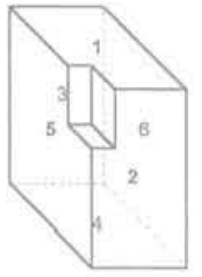

1,2 et 5 sont situés sut les faces de devant

3,4 et 6 sut les faces de deriere

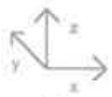

:G. 4 Représentation des différentes faces du massif simulé.

Presentation of different faces of the model

Caractéristiques géotechniques simplifiées du milieu

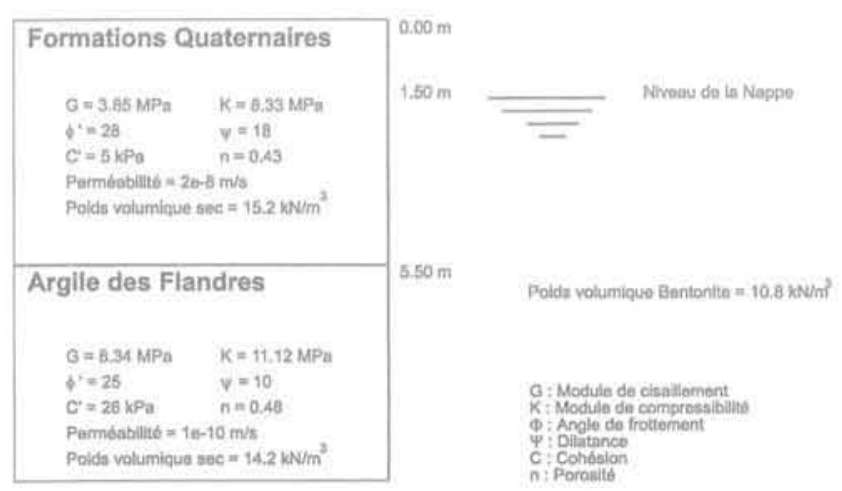

FIG.5 Caractéristiques géotechniques simplifiées du projet.

Simplified geotechnical characteristics of the project.

\section{Description des phases de travaux d'excavation}

La tranchée est creusée sous boue de bentonite. La durée de l'excavation correspond à un poste de travail, à savoir 8 heures (procédure utilisée sur le chantier).

L'excavation est réalisée en huit étapes identiques. Chaque étape est définie de la façon suivante:

- creusement progressif d'un huitième de la hauteur de la tranchée (soit environ $4 \mathrm{~m}$ ) ;

- remplacement progressif des éléments excavés par de la boure de bentonite;

- écoulement de l'eau autorisé dans le milieu pendant une heure.

La présence de boue dans la tranchée est reproduite par l'introduction d'une pression hydrostatique normale au flanc de la tranchée; le poids volumique de la boue de bentonite étant de $10,8 \mathrm{kN} / \mathrm{m}^{3}$.

Le creusement de chaque portion de tranchée est considéré instantané vis-à-vis de l'écoulement de l'eau dans le milieu. Entre chaque nouveau creusement, l'écoulement est autorisé sur une période de une heure.

Dans le paragraphe 3.3, l'évolution dans le temps de la réponse du modèle après remplissage de la tranchée par du béton est étudiée.

\section{1 .4}

\section{Points d'observation de la réponse du modèle}

Le but de ce travail est de permettre l'observation des événements engendrés par l'excavation. Pour ce faire, des points d'observations ont été disposés à des endroits particuliers du maillage. Ils permettent ainsi de visualiser (voir Fig. 6) :

- I'évolution de la contrainte totale aux profondeurs de $10,15,20$ et $25 \mathrm{~m}$;

- l'évolution de la pression interstitielle aux mèmes profondeurs.

De manière plus générale, les gradients de pression interstitielle et de pression totale dans certaines zones intéressantes seront représentés. Il en est de même pour les déplacements latéraux à proximité de la tranchée.

\section{2}

\section{Résultats de la simulation tridimensionnelle}

\section{3.,2:1 \\ Introduction}

Les résultats de la simulation numérique sont présentés de la façon suivante : le massif est d'abord observé dans son ensemble grâce à des coupes dans les plans ( $\mathrm{xOz}$ ) et $(\mathrm{yOz})$. Les graphiques illustrent l'état du milieu à la fin de l'excavation, après 8 heures de travaux.

Ensuite, l'évolution de la pression interstitielle et de la contrainte totale aux points spécifiés dans le paragraphe précédent, sont étudiés. 


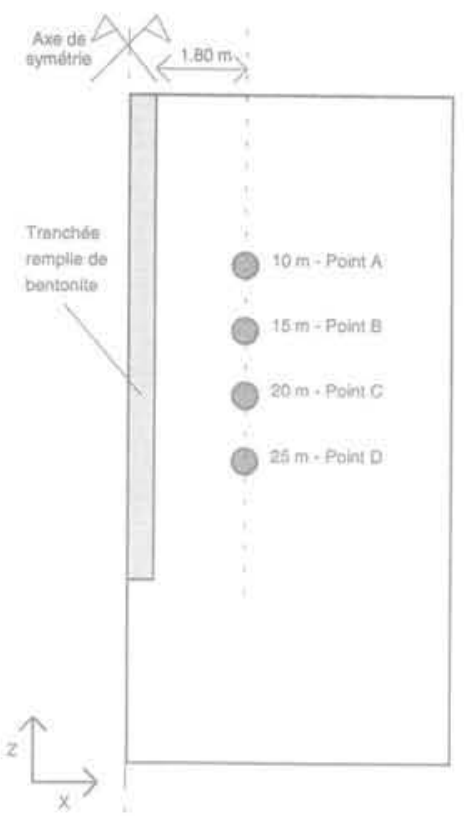

FIG.6 Position des points d'observation de la réponse du modèle.

Location of observation points monitoring model response.

Au paragraphe 3.3, l'attention sera attirée par l'évolution dans le temps de la réponse du modèle, après coulage du béton dans la tranchée.

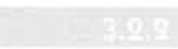

\section{Les déplacements à proximité des flancs de la tranchée}

Les huit étapes du creusement induisent des déplacements du type de ceux représentés sur les figures 8 et 9. A

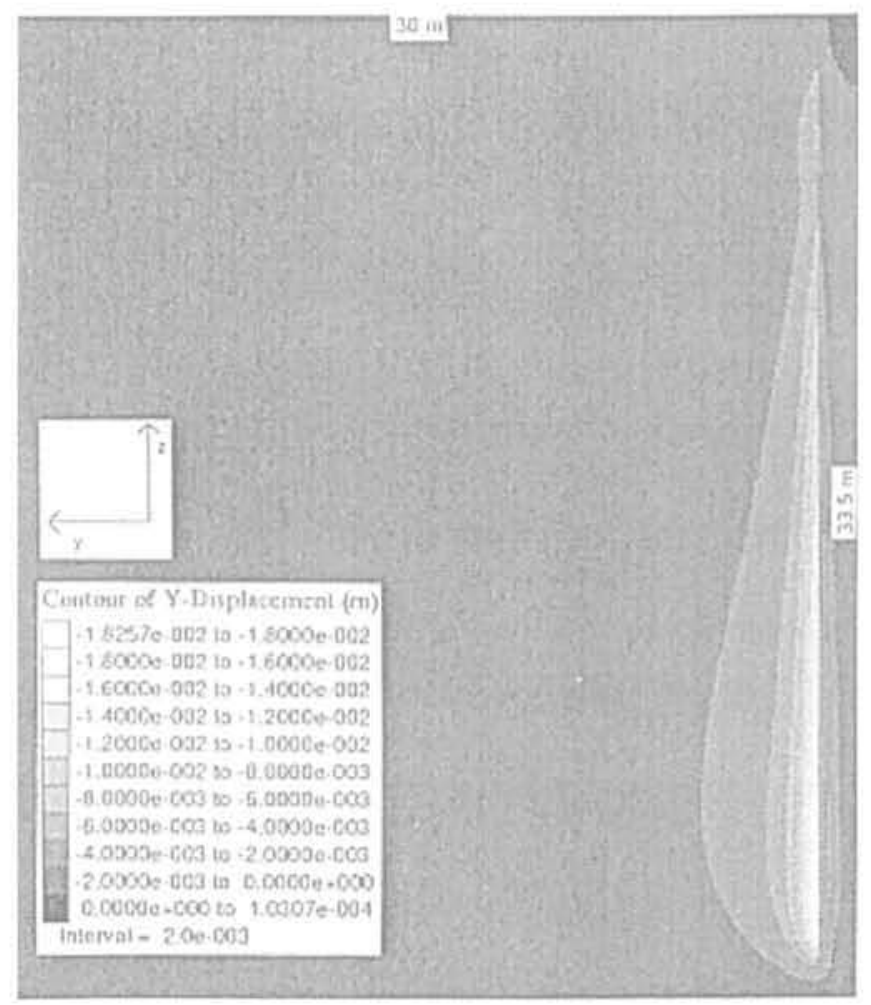

Fig. 8 Déplacements horizontaux dans le plan (yOz).

Horizontal displacements in the (yOz) plane.

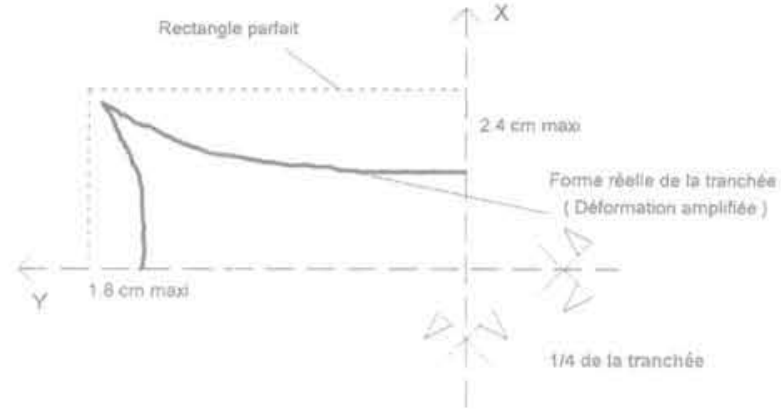

Fig. 7 Représentation de la forme de la tranchée après creusement.

Presentation of the trench shape after excavation.

chaque étape, les mouvements sont toujours plus importants dans le plan vertical $(\mathrm{xOz})$ que dans le plan vertical (yOz). Les maxima atteignent près du fond de fouille $2,4 \mathrm{~cm}$ pour le plan $(\mathrm{xOz})$ et $1,8 \mathrm{~cm}$ dans le plan $(\mathrm{yOz})$. La forme générale de la tranchée, dans tous plans parallèles à ( $\mathrm{xOy}$ ), â la fin de l'excavation correspond à celle de la figure 7 .

Les déplacements dans les formations quaternaires différent légèrement de ceux de la couche d'Argile des Flandres. Ils restent faibles par rapport à l'ensemble.

Remarquons que dans la réalité, le creusement est assuré par un outil qui passe de multiples fois dans la tranchée. Aussi, il n'y a pas de « réduction v visible de la section de la tranchée dans les plans parallèles à (xOy). Cette action, qui n'est sans doute pas neutre visà-vis du comportement du milieu, n'est pas prise en considération dans ce travail.

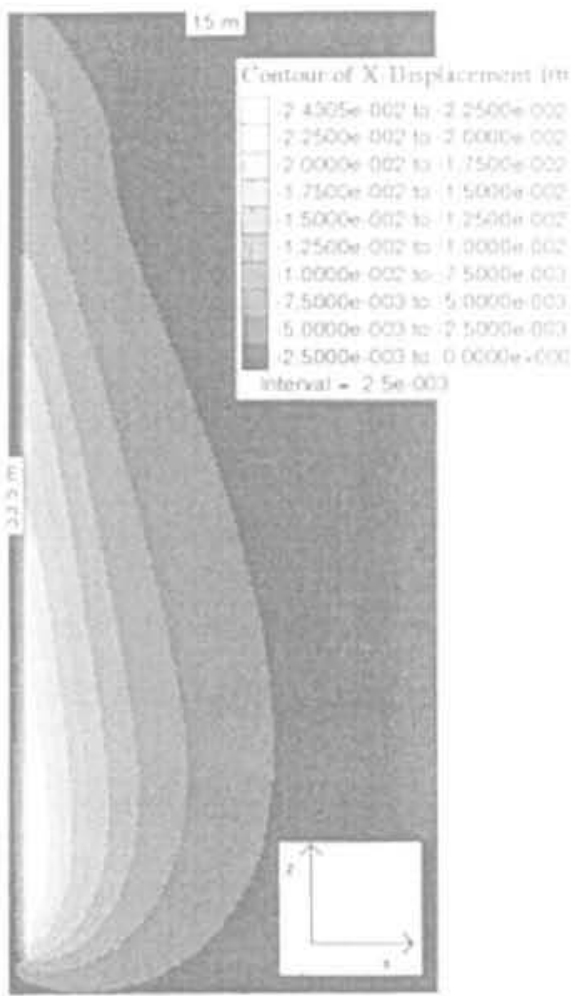

FIG 9 Déplacements horizontaux dans le plan (xOz).

Horizontal displacements in the $(\mathrm{xOz})$ plane, 

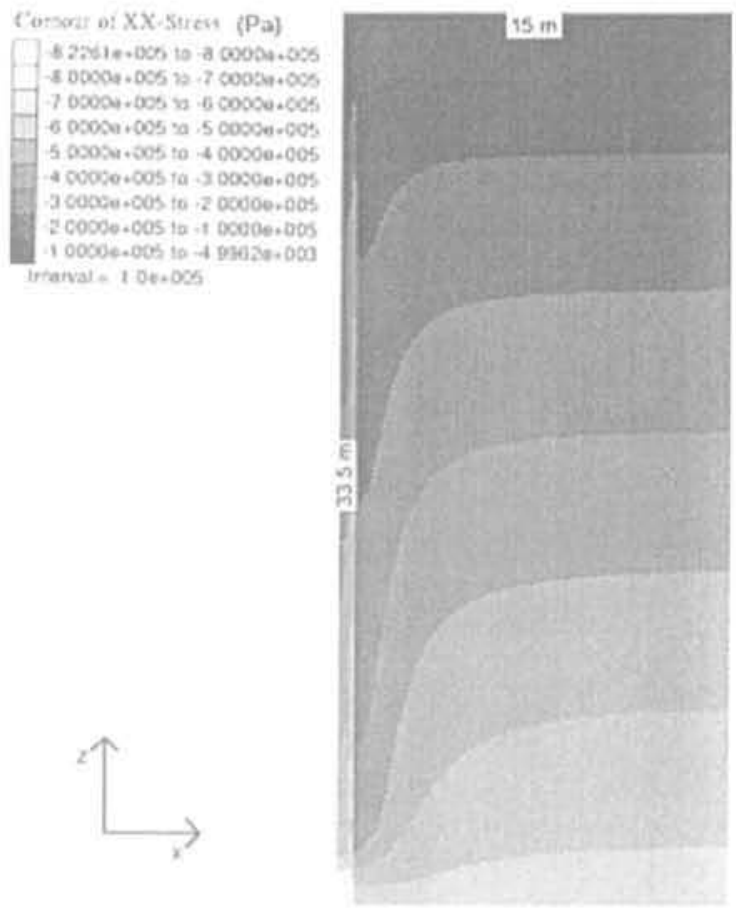

FIG. 10 Répartition des contraintes totales horizontales $\sigma_{\mathrm{n}}$ à la fín de l'excavation (plan xOz).

Distribution of horizontal total stresses $\sigma_{\mathrm{n}}$ at the end of the excavation ( $\mathrm{xOz}$ plane).

\section{$2=3.25$}

\section{Les contraintes totales horizontales et les pressions interstitielles dans le plan $(\mathrm{xOz})$}

Les figures 10 et 11 représentent respectivement la répartition des contraintes totales horizontales $\sigma_{x x}$ et la répartition des pressions interstitielles à la fin de l' excavation (dans le plan $\mathrm{xOz}$ ).

\section{- Les contraintes totales horizontales $\sigma_{x x}$}

Le graphique 10 montre très nettement qu'à la fin de l'excavation de la tranchée, l'état de contrainte totale horizontale $\sigma_{\mathrm{xx}}$ est fortement modifié. L'effet du creusement se fait ressentir jusqu'à une distance d valant environ $5 \mathrm{~m}$. Cela correspond à un rapport $\mathrm{d} / \mathrm{H}$ de 0,15 $(\mathrm{H}=$ hauteur de la tranchée).

La densité de la bentonite étant plus faible que celle du sol, l'état de contrainte généré par la résistance de la boue à la poussée du sol est naturellement plus faible que l'état de contrainte initial. Ceci explique la diminution de $\sigma_{x x}$ à l'approche de la tranchée.

Les contraintes $\sigma_{y y}, \sigma_{z z^{\prime}}, \sigma_{y y} \sigma_{x z^{\prime}} \sigma_{y z}$ et l'état de plasticité du domaine ne sont pas presentés. On notera que seule une zone proche du bord des tranchées est concernee par la plasticité. La majorité du domaine reste élastique.

Remarquons également que la répartition des contraintes $\sigma_{x x}, \sigma_{v y}$ dans les deux plans (xOz) et $(\mathrm{yOz})$ est similaire à celle que l'on rencontre dans le cadre de la réalisation d'un forage cylindrique dans un matériau élastoplastique.

\section{- Les pressions interstitielles}

Le graphique 11 indique une forte décroissance de

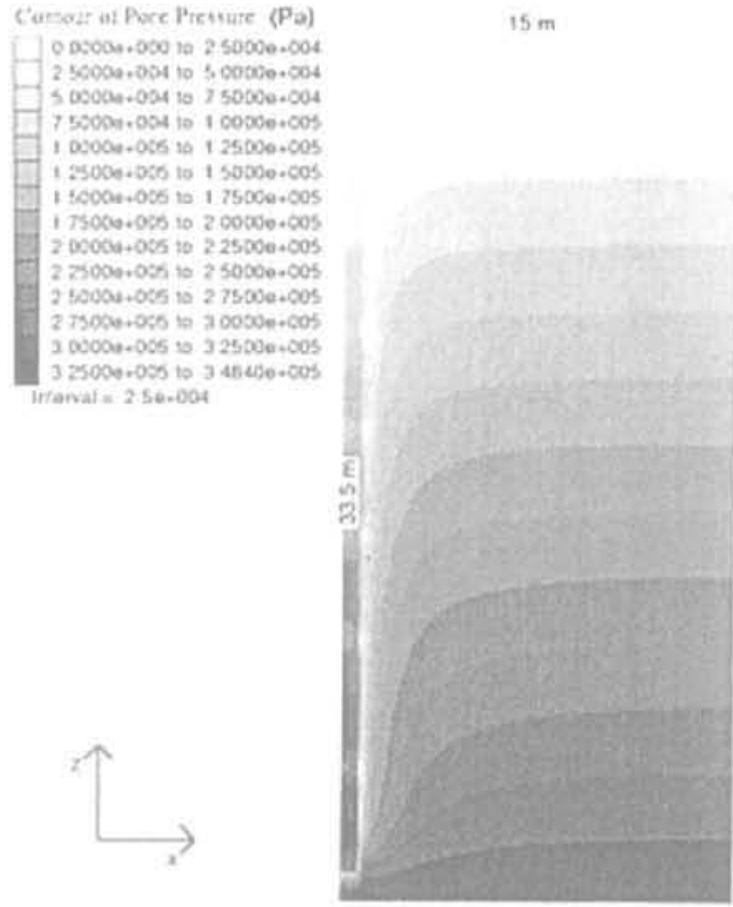

FG.11 Répartition des pressions interstitielles à la fin de l'excavation (plan $\mathrm{xOz}$ ).

Distribution of pore pressures at the end of the excavation ( $\mathrm{xOz}$ plane).

la pression interstitielle à l'approche de la tranchée. On observe d'ailleurs que, juste derrière le bord de la tranchée, la pression est très faible sur une bande de près de $50 \mathrm{~cm}$. Les déplacements horizontaux présentés précédemment sont, sans doute, à l'origine de ce phénomène.

Dans le reste du milieu, et comme pour la contrainte totale, la variation de pression s'étend jusqu'à environ $5 \mathrm{~m}$.

Au sein des formations quaternaires, la pression interstitielle n'évolue pas puisqu'elle a été fixée dès le départ. En tout état de cause, la perméabilité de cette couche est grande vis-à-vis de celle de l'Argile des Flandres, ce qui justifie ce choix.

\section{3iace}

Les contraintes totales horizontales et les pressions interstitielles dans le plan $(\mathrm{yOz})$

Les figures 12 et 13 représentent respectivement la répartition des contraintes totales horizontales $\sigma_{\text {. }}$ et la répartition des pressions interstitielles à la fin de lexcavation (dans le plan yOz).

\section{- Les contraintes totales horizontales $\sigma_{x x}$}

Le graphique 12 montre très nettement qu'à la fin de l'excavation de la tranchée, l'état de la contrainte totale horizontale $\sigma_{\text {z. }}$ est fortement modifié. L'effet du creusement se fait ressentir jusqu'à une distance d valant environ $5 \mathrm{~m}$. Cela correspond à un rapport $\mathrm{d} / \mathrm{H}$ de 0,15 .

Les commentaires liés aux observations effectuées dans le plan $(\mathrm{xOz})$ restent valables dans le plan $(\mathrm{yOz})$. Toutefois, il est nécessaire de considérer les contraintes relativement à la direction du bord de la tranchée. 


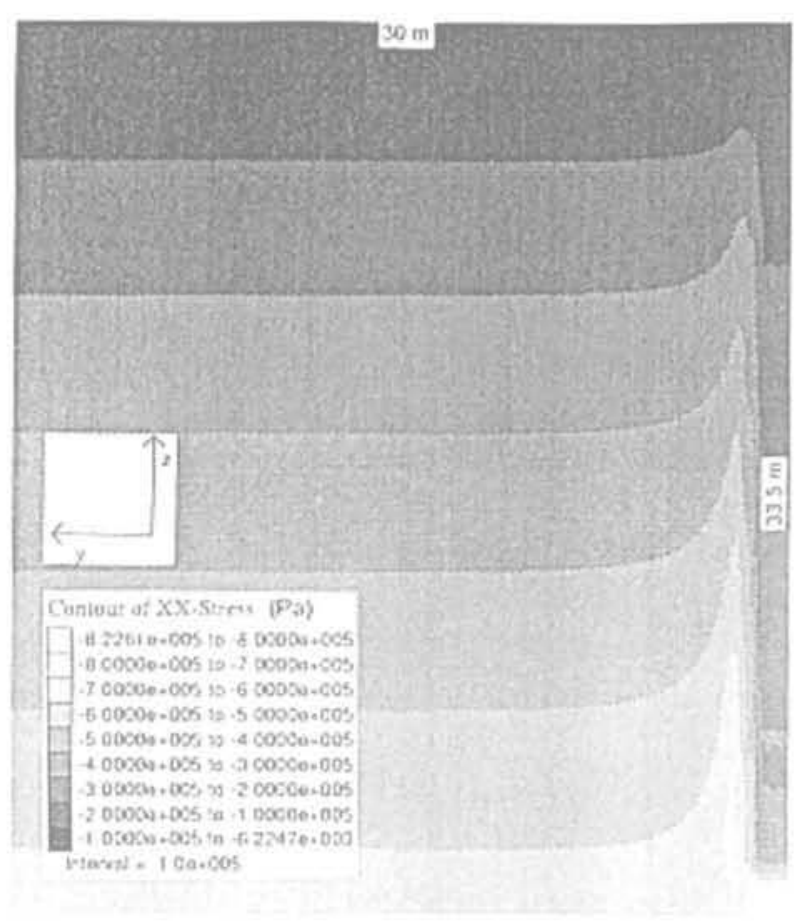

FiG.12 Répartition des contraintes totales horizontales $\sigma_{\mathrm{xx}}$ à la fin de l'excavation (plan yOz).

Distribution of horizontal total stresses $\sigma_{\mathrm{u}}$ at the end of the excavation ( $\mathrm{yOz}$ plane).

Ainsi, les observations effectuées dans le plan (xOz) concernant $\sigma_{x x}$ s'appliquent dans le plan $(y O z)$ à la contrainte $\sigma_{y y}$

Les contraintes $\sigma_{y y^{\prime}} \sigma_{z z^{\prime}} \sigma_{x y^{\prime}} \sigma_{x x^{\prime}} \sigma_{y z}$ ne sont pas présentées.

\section{- Les pressions interstitielles}

Le graphique 13 indique un comportement de la pression interstitielle similaire à celui de la contrainte totale horizontale $\sigma_{\mathrm{yz}}$ dans le plan (yOz). Ce comportement est assez surprenant : la pression interstitielle devrait diminuer à l'approche de la tranchée. Une étude détaillée du phénomène, non décrite dans cet article, a montré que ce comportement est lié à un effet de forme de la tranchée parallélépipédique, venant s'ajouter à l'effet de profondeur et de proximité du pied de la tranchée dans la direction $\mathrm{Oz}$.

Comme pour la contrainte totale, la variation de la pression interstitielle s'étend jusqu'à environ 5 mètres.

Au sein des formations quaternaires, la pression interstitielle n'évolue pas, puisqu'elle a été fixée dès le départ.

\section{5 \\ Évolution de la contrainte totale horizontale aux points marqués (dans le plan $(\mathrm{xOz})$ )}

La figure 14 représente l'évolution de la contrainte totale horizontale aux quatre points A, B, C et D (voir Fig. 6) en fonction du temps. Ce graphique indique que la contrainte à une profondeur $z$ n'est perturbée que lorsque la tranchée atteint et dépasse cette profondeur.

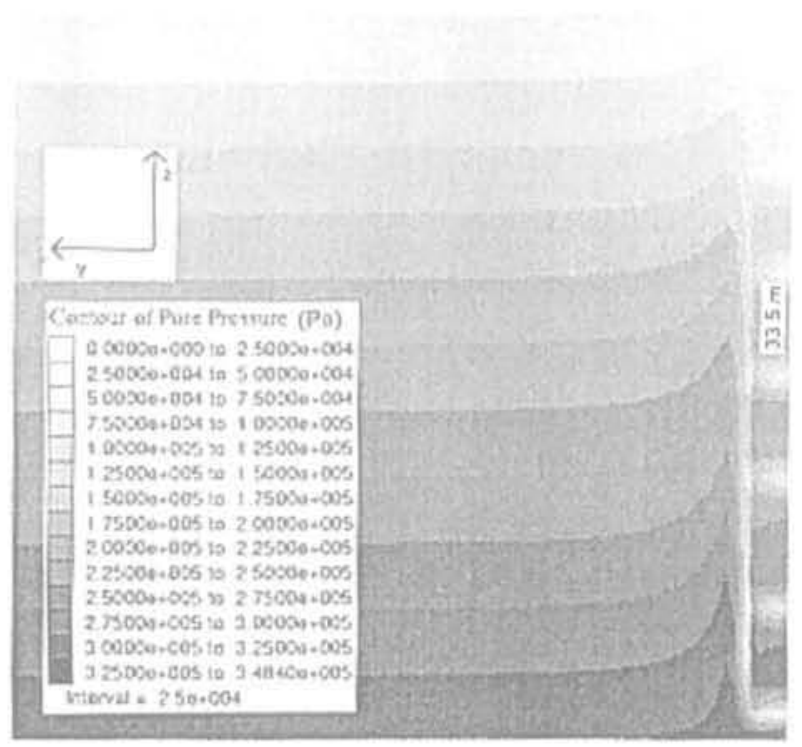

FG. 13 Répartition des pressions interstitielles à la fin de l'excavation (plan yOz).

Distribution of pore pressures at the end of the excavation ( $\mathrm{yOz}$ plane).

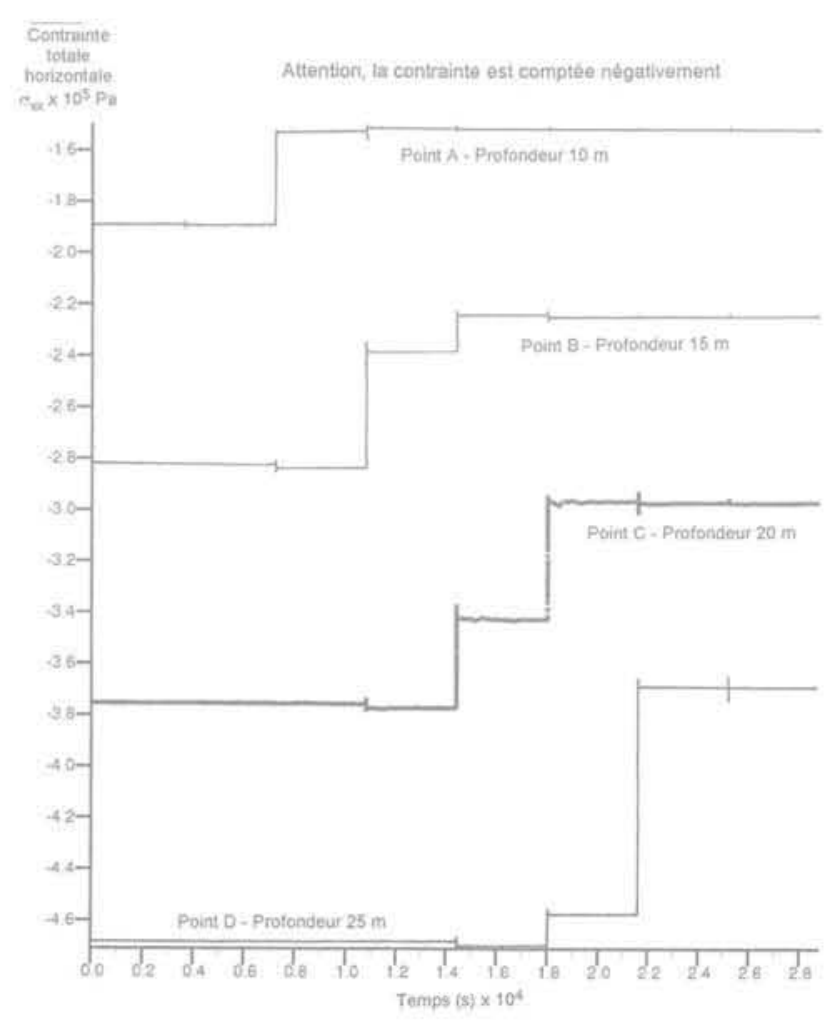

FG. 14 Evolution de la contrainte totale horizontale aux points marqués. Representation of horizontal total stresses, versus time, at labeled points. 
Dès que ladite profondeur est dépassée de 2 ou $3 \mathrm{~m}$, la pression se stabilise et reste constante jusqu'à la fin de l'excavation.

Le tableau suivant indique en pourcentage, la réduction de la contrainte totale en fonction de la profondeur considérée.

TABLEAU1 Réduction de $\sigma_{\text {en }}$ en $\%$, aux points marqués, à la fin de l'excavation. Pourcentage reduction of $\sigma$, at labeled points. at the end of the excavation.

\begin{tabular}{l|c|c|c} 
Profondeur & $\begin{array}{c}\text { Vateur initiale } \\
\mathrm{MPa}\end{array}$ & $\begin{array}{c}\text { Valeur finale } \\
\mathrm{MPa}\end{array}$ & $\begin{array}{c}\text { Réduction } \\
\text { de } \sigma_{x x} \text { en \% }\end{array}$ \\
\hline Point A-10 m & 0,188 & 0,150 & 21 \\
\hline Point B-15m & 0,282 & 0,225 & 20 \\
\hline Point C-20 m & 0,376 & 0,300 & 20 \\
\hline Point D-25m & 0,472 & 0,370 & 22 \\
\hline
\end{tabular}

La contrainte totale horizontale diminue donc d'environ $20 \%$ (aux points marqués) au cours du creusement de la tranchée. Il sera intéressant de voir si ces nouvelles valeurs perdurent après le coulage du béton.

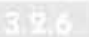

\section{Évolution de la pression interstitielle aux points marqués (dans le plan $(\mathrm{xOz})$ )}

La pression interstitielle aux points marqués se comporte de la même manière que la contrainte totale horizontale (voir Fig. 15). Cette pression n'est perturbée que par le passage de l'outil à la profondeur à laquelle elle est mesurée. Ensuite, elle reste constante jusqu'à la fin du creusement.

Le tableau suivant indique en pourcentage, la réduction de la pression interstitielle u en fonction de la profondeur considérée.

TABLEAU II Réduction de la pression interstitielle en $\%$, aux points marqués, à la fin de Pexcavation.

Pourcentage reduction of pore pressures at labeled points, at the end of the excavation

\begin{tabular}{l|c|c|c} 
Profondeur & $\begin{array}{c}\text { Valeur initiale } \\
\mathrm{MPa}\end{array}$ & $\begin{array}{c}\text { Valeur finale } \\
\mathrm{MPa}\end{array}$ & $\begin{array}{c}\text { Réduction } \\
\text { de u en \% }\end{array}$ \\
\hline Point A-10m & 0,083 & 0,072 & 14 \\
\hline Point B-15 m & 0,132 & 0,114 & 14 \\
\hline Point C-20 m & 0,181 & 0,155 & 14 \\
\hline Point D-25 m & 0,230 & 0,198 & 14 \\
\hline
\end{tabular}

La pression interstitielle diminue donc d'environ $14 \%$ (aux points marqués) au cours du creusement de la tranchée. Quel sera son comportement au cours du temps, après le coulage du béton?

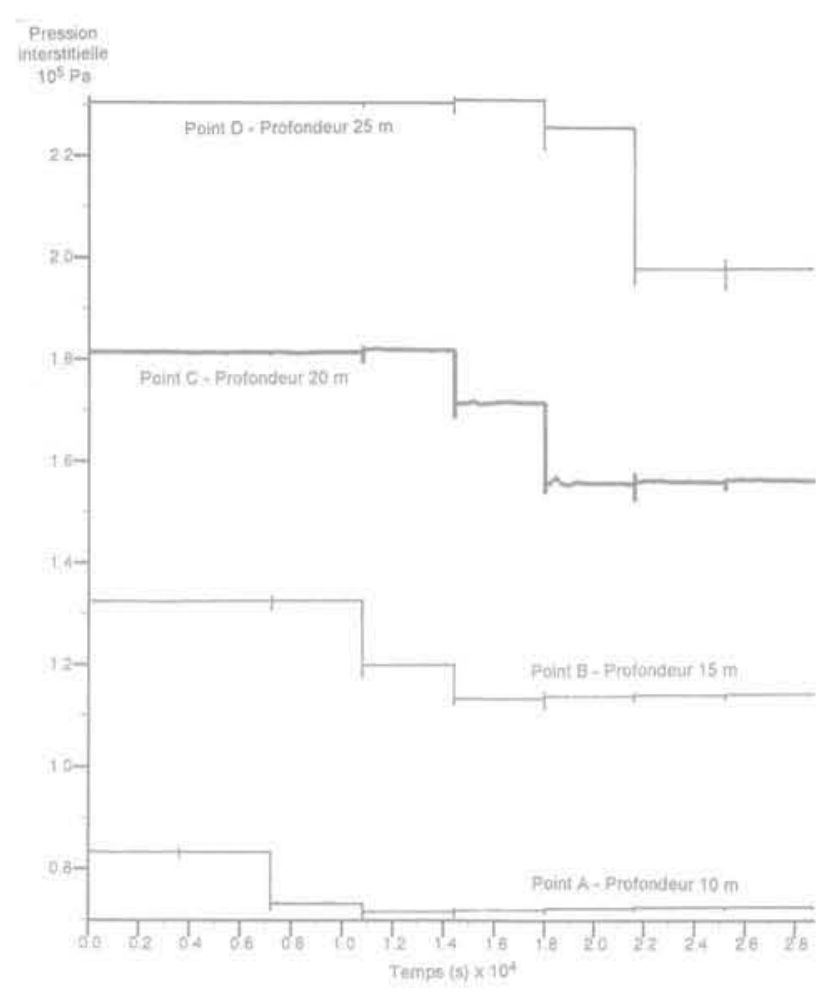

FIG.15 Evolution de la pression interstitielle aux points marqués.

Representation of pore pressures, versus time, at labeled points.

\section{Évolution dans le temps de la réponse du modèle, après remplissage de la tranchée par du béton}

Introduction

L'étape durant laquelle la boue de bentonite contenue dans la tranchée est remplacée progressivement par du béton, ainsi que la phase de prise de celui-ci sont très délicates à modéliser. Trois possibilités au moins s'offrent à nous :

- le béton se comporte comme la bentonite, c'est-à-dire qu'il est liquide. Il génère alors une poussée hydrostatique sur le bord de la tranchée qui est fonction de sa densité, à savoir environ 2,3. La densité du sol en place étant de l'ordre de 2 , le béton devrait (c repousser ») le sol... - la mise en place et la prise du béton sont instantanés à T'échelle de l'ouvrage. Les déplacements des bords de la paroi sont alors fixés :

- le comportement est intermédiaire. Le béton est un fluide de propriétés telles que la poussée exercée sur le bord de la tranchée n'est pas hydrostatique. En outre, ses propriétés changent au fur et à mesure de la prise.

La solution que nous retenons pour cette étude est la seconde. Notre choix est motivé par les raisons suivantes : - les mesures inclinométriques réalisées sur un chantier similaire (chantier Tourcoing-Centre) n'ont pas 


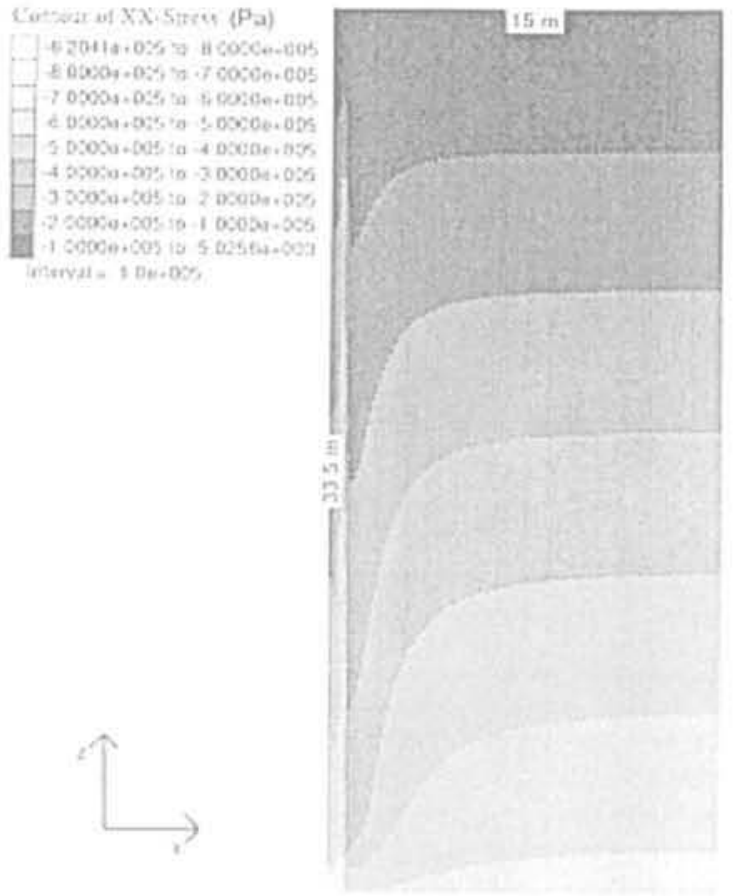

FiG. 16 Répartition des contraintes totales horizontales $\sigma_{x \alpha}$. Évolution dans le temps (plan xOz).

Representation of horizontal total stresses $\sigma_{2}$ versus time ( $\mathrm{xOz}$ plane)

révélé de recul du sol durant le bétonnage. Aussi, le béton ne se comporte pas comme un simple liquide; la proposition 1 est donc écartée :

- la troisième possibilité est la plus probable mais, délicate et complexe, elle introduit des incertitudes qui rendraient l'interprétation de ce travail encore plus difficile.

La seconde hypothèse simplificatrice est donc retenue. Simple à mettre en œuvre, elle devrait permettre d'observer l'évolution dans le temps de la réponse du modèle.

Cette évolution dans le temps du modèle a donc été simulée. La période représentée est d'environ trois mois, ce qui correspond globalement à l'intervalle de temps séparant le bétonnage du panneau de la paroi moulée, des premiers travaux d'excavation de la station de métro.

Seul le plan (xOz) nous intéresse, compte tenu du fait que c'est le plan perpendiculaire à la future paroi moulée. Par ailleurs, dans le laps de temps considéré, d'autres panneaux ont été réalisés, ce qui rend l'observation des informations dans le plan (yOz) sans réel intérêt.

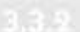

\section{Les contraintes totales horizontales et les pressions interstitielles dans le plan ( $\mathrm{xOz}$ )}

Les graphiques 16 et 17 sont à comparer aux figures 10 et 11.

\section{- Les contraintes totales horizontales $\sigma_{x x}$}

La figure 16 révèle que la contrainte totale horizontale n'évolue que très peu, durant la période des trois

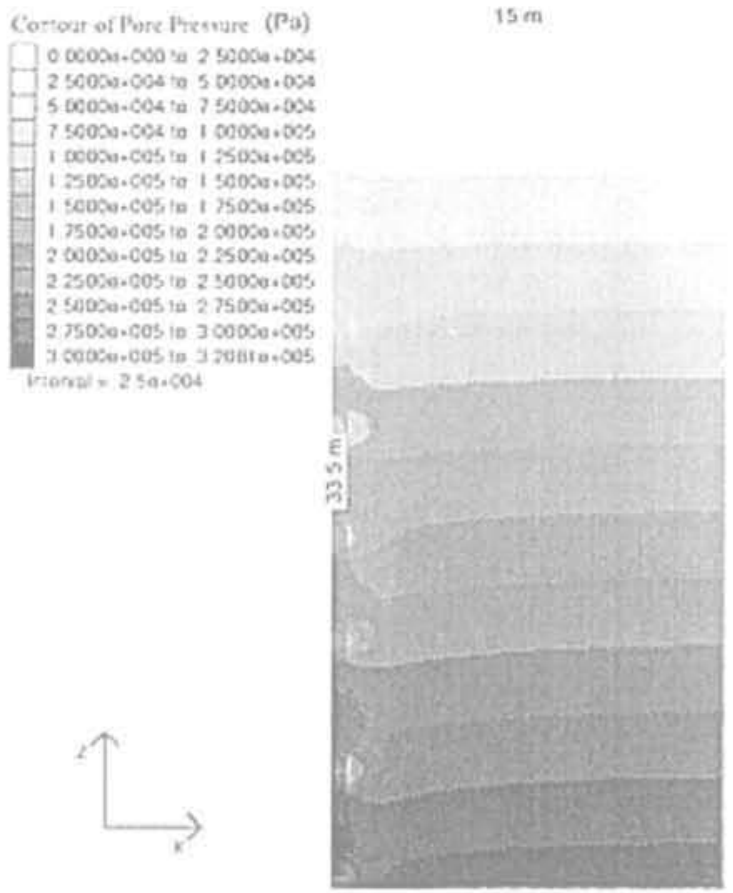

FIG. 17 Répartition des pressions interstitielles. Evolution dans le temps (plan xOz). Representation of pore pressures versus time (xOz plane).

mois. Les deux graphiques 10 et 16 sont pour ainsi dire superposables, La figure 18 permet de se rendre compte du phénomène aux points marqués, en fonction du temps.

\section{- Les pressions interstitielles}

La répartition des pressions interstitielles, représentées sur la figure 17 est très différente de celle de la figure 11. Sur l'ensemble du massif, la pression interstitielle remonte de façon à « revenir » vers un état quasi initial. Pourtant, la période de trois mois n'est pas suffisante pour atteindre cet état initial. La vitesse de ce phénomène est visible sur le graphique 19.

\section{zikn \\ Évolution de la contrainte totale horizontale aux points marqués}

La figure 18 représente l'évolution de la contrainte totale horizontale aux points A, B, C et D en fonction du temps. L'augmentation moyenne des valeurs pour les quatre courbes, sur les trois mois, est inférieure à $4 \%$.

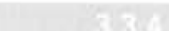

\section{Évolution de la pression interstitielle aux points marqués}

La figure 19 représente l'évolution de la pression interstitielle aux points marqués $A, B, C$ et $D$ en fonction du temps. Le tableau III indique en pourcentage, l'augmentation de la pression interstitielle, en fonction de la profondeur considérée, par rapport aux valeurs obtenues à la fin du creusement de la tranchée. 


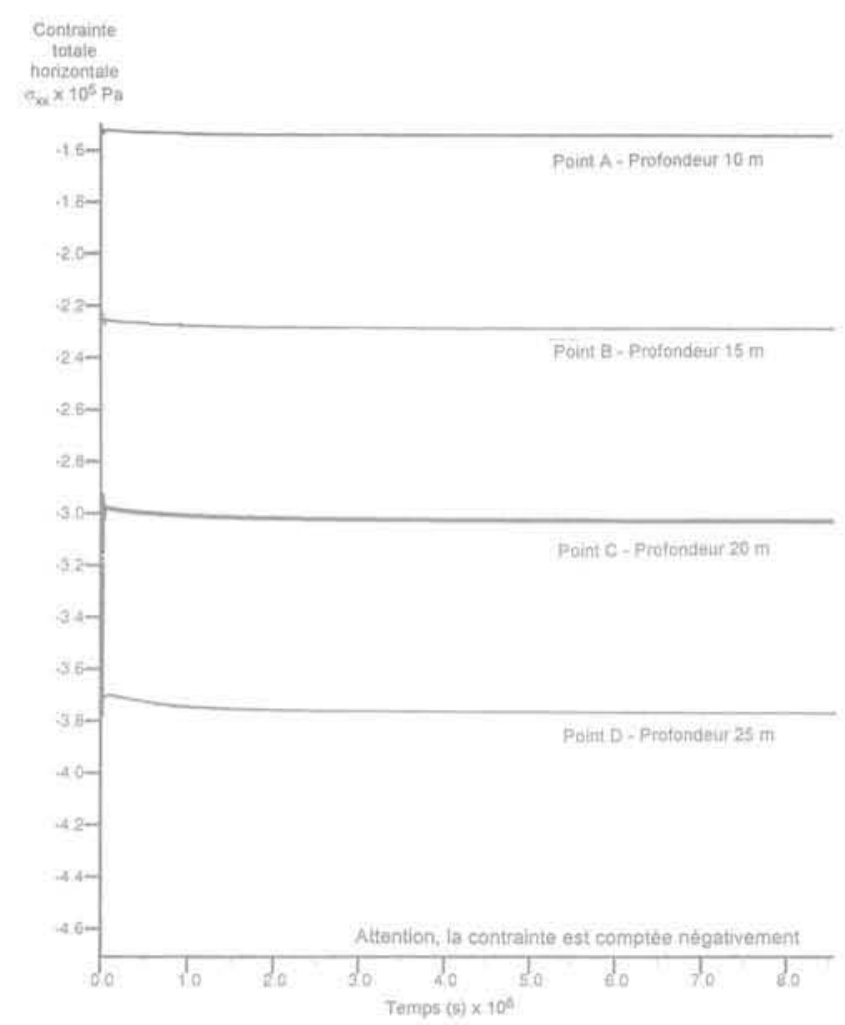

FIG. 18 Évolution de la contrainte totale horizontale aux points marqués. Évolution dans le temps.

Evolution of horizontal total stresse $\sigma_{\mathrm{x}}$ at labeled points, versus time.

TABLEAU III Augmentation de la pression interstitielle en $\%$, aux points marqués, par rapport à la fin du creusement.

Increase pouncentage of pore pressures, at labeled points. Reference time: end of excavation.

\begin{tabular}{l|c|c|c} 
Profondeur & $\begin{array}{c}\text { Après } \\
\text { creusement } \\
\text { MPa }\end{array}$ & $\begin{array}{c}\text { Après } \\
\text { creusement } \\
+3 \text { mois } \\
\text { (MPa) }\end{array}$ & $\begin{array}{c}\text { Augmentation } \\
\text { de u en \% }\end{array}$ \\
\hline Point A-10m & 0,072 & 0,080 & 11 \\
\hline Point B-15 m & 0,114 & 0,127 & 11 \\
\hline Point C-20 m & 0,155 & 0,172 & 11 \\
\hline Point D-25 m & 0,198 & 0,219 & 11 \\
\hline
\end{tabular}

TABLEAUIV Réduction de la pression interstitielle en $\%$, aux points marqués, entre l'état initial et l'état actuel.

Decrease pourcentage of pore pressures.

at labeled points; between initial state and

3 months after excavation.

\begin{tabular}{|c|c|c|c}
\hline Profondeur & $\begin{array}{c}\text { Avant } \\
\text { creusement } \\
\mathrm{MPa}\end{array}$ & $\begin{array}{c}\text { Aprés } \\
\text { creusement } \\
+3 \text { mois } \\
\text { (MPa) }\end{array}$ & $\begin{array}{c}\text { Réduction } \\
\text { de uen \% }\end{array}$ \\
\hline Point A-10m & 0,083 & 0,080 & 4 \\
\hline Point B-15 m & 0,132 & 0,127 & 4 \\
\hline Point C- $20 \mathrm{~m}$ & 0,181 & 0,172 & 5 \\
\hline Point D-25 m & 0,230 & 0,219 & 5 \\
\hline
\end{tabular}

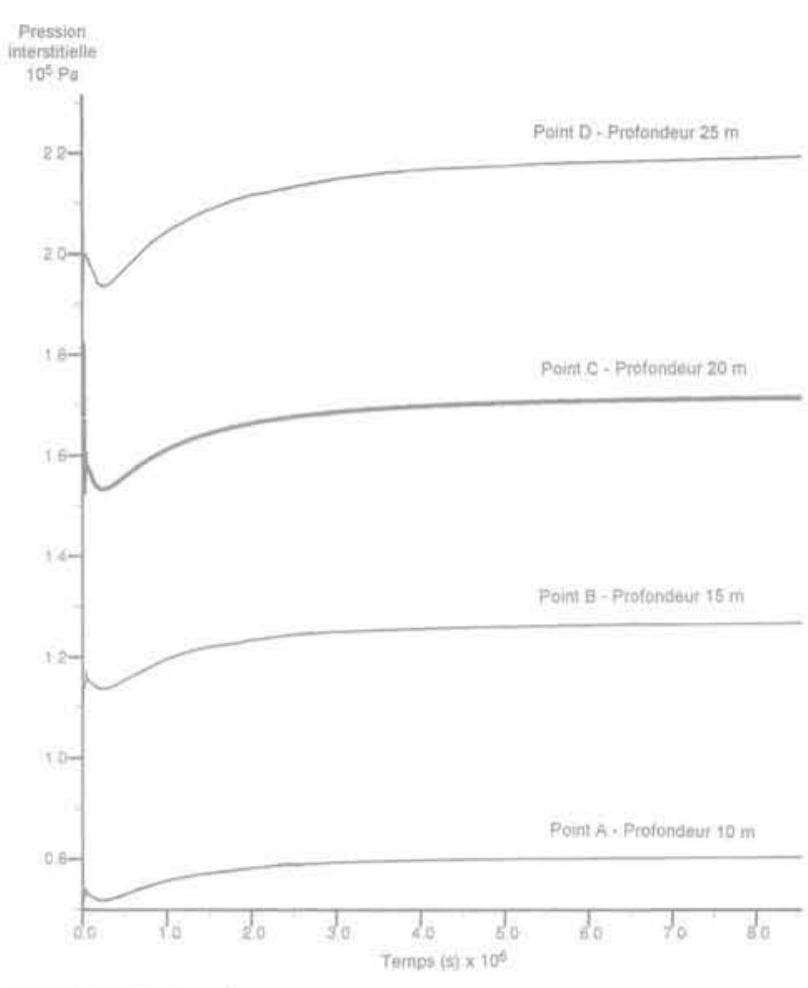

FIG. 19 Évolution de la pression interstitielle aux points marqués. Évolution dans le temps. Representation of pore pressure, at labeled points, versus time.

Au total, la réduction de la pression interstitielle entre l'état initial et l'état actuel est indiquée dans le tableau IV :

Pour un observateur qui se contenterait de comparer la pression interstitielle avant creusement de la tranchée sous boue de bentonite avec celle existant après trois mois de bétonnage, il apparaitrait que la pression interstitielle est peu perturbée.

\section{$3=15$ \\ Conclusion}

Le cumul des deux actions suivantes:

- le creusement sous boue de bentonite de la tranchée; - la mise en place du béton (et la phase d'attente des étapes ultérieures), a pour effets dans la simulation 3D réalisée:

- une diminution, puis une restabilisation partielle des pressions interstitielles ;

- une diminution conséquente de la contrainte totale horizontale, et donc de la contrainte horizontale effective, compte tenu de l'évolution de la pression interstitielle.

\section{4}

\section{Synthèse}

La simulation numérique tridimensionnelle biphasique totalement couplée de la réalisation d'une tranchée sous boue de bentonite du chantier ALMA-GARE conforte les résultats de la littérature. Nous avons effecti- 
vement constaté, dans la simulation, que le processus de l'installation d'une paroi moulée dans une argile cause: - des déplacements dans le milieu environnant; - des réductions conséquentes et durables des contraintes horizontales totales et effectives.

Les phénomènes mis en jeu au niveau des déplacements sont les suivants : chaque nouvelle étape de creusement génère, près du pied de la tranchée, des déplacements horizontaux « proportionnels » à la profondeur de creusement. Pourtant, ces mouvements ne semblent pas (ou peu) affecter les parties directement supérieures de la tranchée. C'est comme si un cadre retenait les flancs de la partie supérieure précédemment excavée. Les déplacements de la partie inférieure sont alors assez peu influencés par le reste du modèle. L'excavation produit donc une modification locale des déplacements.

Les mêmes remarques peuvent être formulées à propos des contraintes totales horizontales et des pressions interstitielles. On assiste à une ( localisation $)$ de l'effet créé par le creusement. Cette localisation s'applique d'ailleurs aux trois directions de l'espace puisque la perturbation horizontale du milieu reste confinée à la proximité de la tranchée.

La forme et les dimensions de la tranchée $(\mathrm{L} \times \mathrm{I} \times \mathrm{H})$ jouent un rôle important, compte tenu du fait que le comportement est différent entre le petit et le grand côté de la tranchée. Pourtant, il ne faut pas oublier que les deux zones situées de part et d'autre des petits côtés seront excavées lors du creusement des panneaux secondaires. Par ailleurs, après le remplissage par du béton et la prise de celui-ci, le grand côté assurera le rôle mécanique de maintien des terres.

Dans cette optique, on a montré que l'évolution dans le temps du comportement du modèle s'accompagne d'une tendance à la restabilisation des pressions interstitielles (à long terme) mais, que la contrainte totale horizontale n'évolue que très peu. Cette constatation permet d'avancer l'hypothèse que le panneau (isolé) de la paroi moulée se trouverait, après sa phase de mise en place, moins sollicité que l'on ne le supposait dans l'hypothèse du ( tout en place »). De même, la contrainte horizontale effective se trouve réduite près de la tranchée.

\section{4}

\section{Confrontation avec des mesures de chantier}

\section{1}

\section{Les déplacements}

Dans la simulation tridimensionnelle, les déplacements sont locaux et restent liés à chaque incrément de perturbation, en n'affectant que très peu le reste du massif.

\subsection{1}

\section{Observation d'un cas concret}

L'observation d'un cas concret peut donner une idée du comportement réel du sol. Mais, la rareté des données concernant cette phase particulière, jugée " sans effet » par certains auteurs, rend plus difficile ce travail. Notre analyse se basera sur une instrumentation installée sur le chantier Tourcoing-Centre du métro de Lille (ligne 2, lot 2, 1995).

\section{- Le métro de Lille : station Tourcoing-Centre}

Les relevés effectués sur ce chantier permettent d'avoir une idée de l'amplitude des mouvements générés par le creusement d'une des tranchées de la paroi moulée. La figure 20 représente le relevé inclinométrique correspondant (inclinomètre placé à $1 \mathrm{~m}$ du dos de la paroi moulée). Le déplacement maximal est de l'ordre de $1 \mathrm{~cm}$ vers l'intérieur de la station, ce qui correspond à une valeur horizontale $\delta / \mathrm{H}$ de $4.10^{-4}$.

La forme des déplacements correspond à celle obtenue à partir de l'approche tridimensionnelle. Le maximum se situe près du fond de la tranchée.

Il faut noter que la présence d'un ouvrage fondé sur pieux à proximité de la tranchée peut modifier les déplacements du fait du rôle de rigidification du massif par l'ensemble pieux-bâtiment. Par ailleurs, l'intervalle de tolérance des mesures, en cumulé, est relativement important.

\section{1 .2}

\section{Conclusion concernant les déplacements}

Le chantier Tourcoing-Centre a été instrumenté dans le but d'observer les déplacements de l'écran et des ouvrages avoisinants. Les informations issues des relevés inclinométriques sont à ce titre très importantes. Elles montrent que les résultats de l'approche 3D sont cohérents.

Aussi, nous pouvons raisonnablement penser, dans l'état actuel de nos connaissances, que l'approche tridimensionnelle semble assez bien prédire les déplacements générés par la réalisation des tranchées, sous boue de bentonite.

Des mesures réalisées sur d'autres chantiers, seraient toutefois nécessaires, pour confirmer la validité, dans le domaine de la prédiction des déplacements, de l'approche tridimensionnelle biphasique couplée.

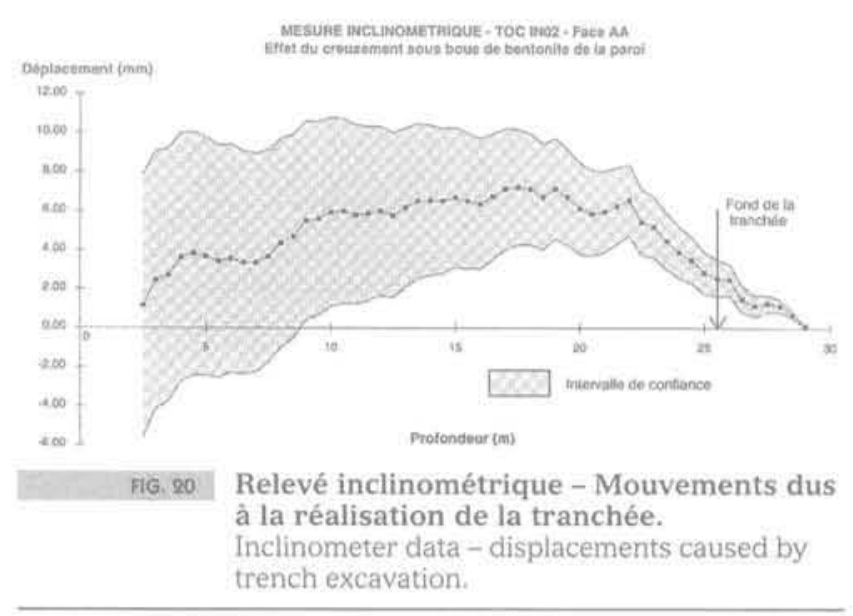




\section{Les contraintes totales horizontales}

\subsection{2.}

\section{Introduction}

L'approche tridimensionnelle montre une décroissance significative de l'état de contraintes totales horizontales à l'approche de la tranchée. La baisse de contrainte est importante et rapide.

\section{29.9.}

\section{Confrontation avec le chantier Alma-Gare}

Des mesures de la contrainte totale horizontale ont été réalisées dans des forages disposés exactement aux points marqués A, B, C et D (voir Fig. 6). Bien qu'aucune mesure n'ait été effectuée dans la période de réalisation de la tranchée sous boue de bentonite, nous disposons de valeurs relevées avant les premières étapes d'excavation de la station de métro.

Le tableau $V$ regroupe les différentes estimations, ainsi que les mesures de $\sigma_{x x}$. On y trouve:

- colonne 2 ; une estimation des valeurs des contraintes initiales, en aval de tous travaux:

- colonne 3 : les mesures réalisées en forage :

- colonne 4 : les résultats du calcul 3D.

TABLEAUV Synthèse des différentes estimations de la contrainte totale horizontale.

Summary of computed and in situ horizontal total stresses.

\begin{tabular}{l|c|c|c}
$\begin{array}{c}\text { Point et } \\
\text { profondeur }\end{array}$ & $\begin{array}{c}\text { Contrainte } \\
\text { initiale } \\
\text { (MPa) }\end{array}$ & $\begin{array}{c}\text { Mesure } \\
\text { in situ } \\
\text { (MPa) }\end{array}$ & $\begin{array}{c}\text { Calcul } \\
3 \mathrm{D} \\
(\mathrm{MPa})\end{array}$ \\
\hline Point A-10 m & 0,190 & 0,100 & 0,150 \\
\hline Point B-15 m & 0,280 & 0,195 & 0,225 \\
\hline Point C-20 m & 0,380 & 0,180 & 0,300 \\
\hline Point D-25 m & 0,470 & 0,380 & 0,370 \\
\hline
\end{tabular}

Les valeurs mesurées sur le terrain sont très inférieures à celles estimées comme étant les valeurs initiales.

Les résultats issus du calcul 3D sont meilleurs, mais l'écart par rapport aux mesures in situ n'est pas négligeable. Ainsi, les prédictions proposées pour les profondeurs de $10 \mathrm{~m}$ et $20 \mathrm{~m}$ sont assez mauvaises, alors que celles de $15 \mathrm{~m}$ et $25 \mathrm{~m}$ sont acceptables.

\section{0 .}

\section{Conclusion concernant la contrainte horizontale totale}

Pour le chantier Alma-Gare, les mesures disponibles ne sont pas réalisées dans les meilleures conditions pour tirer des conclusions irrévocables. L'intérêt de l'époque pour ces informations n'était pas concor- dant avec celui de cette étude. Les relevés n'ont donc pas été effectués durant la phase qui nous intéresse. Ainsi privés de mesures initiales, nous ne disposons pas de référence permettant de juger de la réelle décroissance de la contrainte sur le site, ni de son amplitude...

Nonobstant cette remarque, si l'on exclut la mesure effectuée au niveau du point C (graphique 21), la simulation 3D prédit moyennement bien les valeurs mesurées sur le site.

\section{3}

\section{Les pressions interstitielles}

\subsection{1 \\ Introduction}

Comme pour les contraintes totales horizontales, l'approche tridimensionnelle aboutie à une décroissance significative de la pression interstitielle à l'approche de la tranchée, puis une remontée vers l'état initial.

\section{Confrontation avec le chantier Alma-Gare}

Des mesures de la pression interstitielle ont été réalisées dans des forages disposés aux points marqués $\mathrm{A}$, B, C et D (voir Fig. 6). Bien qu'aucune mesure n'ait été effectuée dans la période de réalisation de la tranchée sous boue de bentonite, nous disposons de valeurs relevées avant les premières étapes d'excavation de la station de métro. Il s'agit donc des valeurs obtenues bien après la mise en place et la prise du béton dans la tranchée.

Le tableau VI regroupe les différentes estimations, ainsi que les mesures de la pression interstitielle. On y trouve :

- colonne 2 : une estimation des valeurs des pressions interstitielles, en aval de tous travaux :

- colonne 3 : les mesures réalisées en forage ;

- colonne 4: les résultats du calcul 3D.

La simulation 3D prédit une augmentation de la pression interstitielle au cours des trois mois séparant la réalisation de la paroi, des premières excavations de la station.

TABLEAUVI Synthèse des différentes estimations de la pression interstitielle.

Summary of computed and in situ pore pressures.

\begin{tabular}{l|c|c|c}
$\begin{array}{c}\text { Point et } \\
\text { profondeur }\end{array}$ & $\begin{array}{c}\text { Pression } \\
\text { interstitielle } \\
\text { (MPa) }\end{array}$ & $\begin{array}{c}\text { Mesure } \\
\text { in situ } \\
\text { (MPa) }\end{array}$ & $\begin{array}{c}\text { Calcul } \\
\text { 3D } \\
\text { (MPa) }\end{array}$ \\
\hline Point A-10m & 0,083 & 0,079 & 0,080 \\
\hline Point B-15 m & 0,132 & 0,132 & 0,127 \\
\hline Point C-20m & 0,181 & 0,180 & 0,172 \\
\hline Point D-25 m & 0,230 & 0,222 & 0,219 \\
\hline
\end{tabular}

14 Calcul intégrant le bétonnage et l'attente de trois mois, 3D 


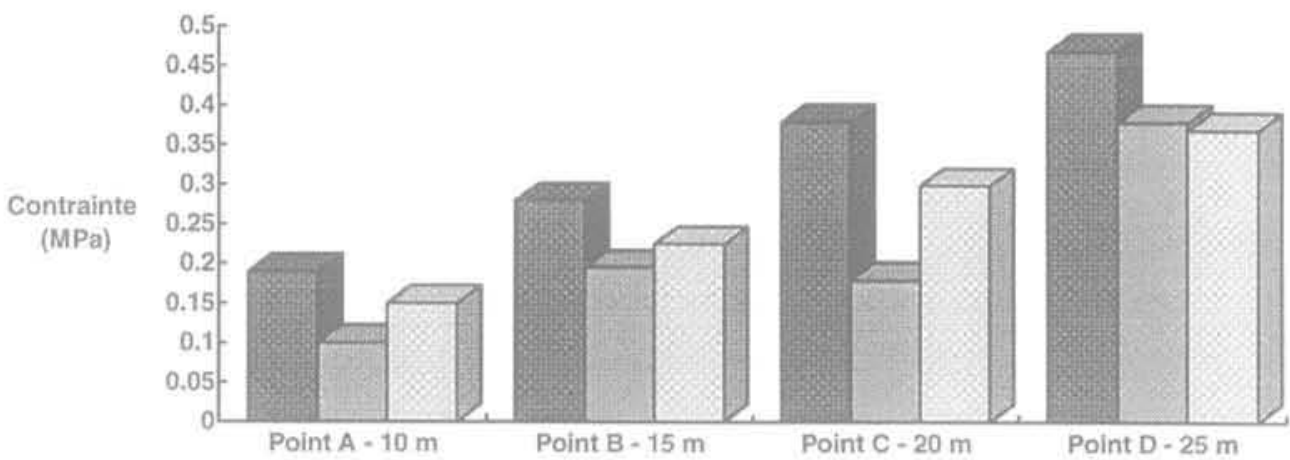

든 contrainte initiale $\square$ Mesure in situ $\square$ Calcul $3 D$

FiG. 21 Représentation des différentes estimations de la contrainte totale horizontale.

Representation of computed and in situ horizontal total stresses.

L'augmentation de la pression n'est pas visible sur les mesures in situ compte tenu du fait qu'aucune mesure n'a eu lieu durant cette phase. Pourtant, les valeurs relevées juste avant les premières étapes d'excavation de la station devraient représenter la réalité en terme de pression. Ce sont ces valeurs que nous avons comparées aux calculs.

\section{4}

\section{Conclusion concernant la pression interstitielle}

Les mesures ont été menées à des dates et dans des conditions qui ne nous permettent pas d'apporter une conclusion sans appel. Pourtant, l'évolution de la pres: sion interstitielle semble relativement bien reproduite par la simulation numérique tridimensionnelle du continuum élastoplastique.

\section{4}

\section{Conclusions et perspectives}

L'absence de mesures réalisées dans des conditions spécifiquement étudiées pour cette étape particulière, rend toute conclusion sujette à controverse. Il est pourtant nécessaire de faire le point sur les résultats de ce travail et de prendre parti en se basant sur les informations dont nous disposons.

Le tableau suivant regroupe les différents arguments en faveur de l'approche tridimensionnelle.

Compte tenu de toutes les remarques que nous avons pu formuler jusqu'à présent, l'approche tridimensionnelle apparaît refléter assez bien le comportement supposé réel du milieu, durant la réalisation d'un panneau de paroi moulée.

Pourtant, cette méthode nécessite des moyens de calculs relativement lourds ${ }^{(1)}$. Il serait donc intéressant

(1) Duree $=7$ jours sur un PC Pentium $90 \mathrm{MHz}$. L'augmentation de la puissance des calculateurs favorisera sans aucun doute la vulgarisation des calculs tridimensionnels.
TABLEAUVII Arguments en faveur de l'approche 3D. Advantages of the 3D method.

\begin{tabular}{c|c}
\hline Estimation des déplacements & $\begin{array}{c}\text { Arguments en faveur } \\
\text { del'approche 3D }\end{array}$ \\
$\begin{array}{c}\text { Eestimation } \\
\text { de la répartition } \\
\text { de Tourcoing-Centre } \\
\text { Réalisme physique }\end{array}$ \\
\hline $\begin{array}{c}\text { Estimation de la répartition } \\
\text { de la pression interstitielle }\end{array}$ & Mesures de Alma-Gare \\
\hline
\end{tabular}

d'employer une approche bidimensionnelle non conventionnelle qui, intégrant une méthodologie particulière, permettrait de s'affranchir de la simulation 3D.

Une approche axisymétrique biphasique couplée, ainsi que son application, ont été étudiées. Elles ne figurent pas dans le présent article.

\section{5}

\section{Conclusion}

La simulation numérique tridimensionnelle biphasique couplée de la réalisation d'une tranchée sous boue de bentonite conforte les résultats de la littérature. Le processus de l'installation d'un panneau de paroi moulée dans une argile raide cause, d'une part, des déplacements non négligeables dans le milieu environnant, d'autre part, des réductions conséquentes et durables des contraintes horizontales totales et effectives.

Chaque étape de creusement de la tranchẻe est à l'origine de phénomènes localisés; qu'il s'agisse des déplacements ou des variations de contraintes. La forme de la tranchée conditionne la réponse de certains facteurs, comme la pression interstitielle par exemple. 


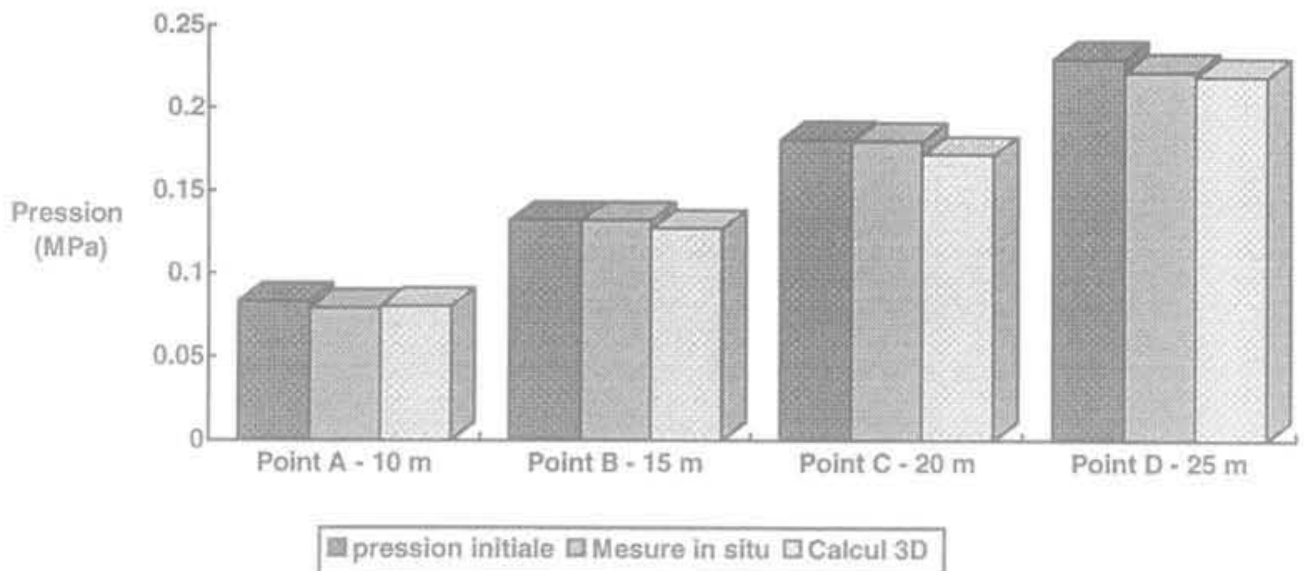

FG. 22 Représentation des différentes estimations de la pression interstitielle. Representation of computed and in situ pore pressures.

Par ailleurs, on a montré que l'évolution dans le temps du comportement du modèle s'accompagne d'une tendance à la restabilisation des pressions interstitielles. Mais, la contrainte totale horizontale n'évolue pas sur la période séparant le bétonnage du panneau, des premiers travaux d'excavation de la station de métro.

La confrontation des résultats de l'approche 3D avec des mesures et des observations des sites de Alma-Gare, de Tourcoing-Centre et même de « Bell Common Tunnel ) (non présenté) permet de conclure que la simulation tridimensionnelle reflète assez correctement le comportement supposé réel du milieu.

Compte tenu des lourds moyens de calcul qu'il est nécessaire de déployer pour une résolution 3D, une approche axisymétrique biphasique couplée pourra être envisagée. Enfin, la réalisation complète d'une paroi moulée nécessite la mise en ceuvre de plusieurs panneaux juxtaposés. Les phénomènes rencontrés dans le cadre de la réalisation d'un panneau sont-ils extrapolables à $n$ panneaux?
REMERCIEMENTS

Cet article est la publication n $n^{\circ} 97005$ du BRGM. II a été réalisé dans le cadre d'un projet co-financé par le BRGM (Contrat $n^{\circ}$ 9410001) et la Région Nord-Pas-de-Calais. L'auteur remercie H. Modaressi (BRGM) et J.-F. Ouvry (ANTEA) pour leur participa- tion active et leurs nombreux conseils, L'auteur remercie également la société ITASCA pour le prẻt du loqiciel FLAC 3D quia permis les modélisations numériques présentées dans cet article.

\section{Bibliographie}

Billaux D., Cundall P. - Simulation des géomatériaux par la méthode des éléments Lagrangiens. Revue française de Géotechnique, 1993, $n^{\circ}$ 63, pp. 9-21.

Cundall P. - Notice du logiciel FLAC ITASCA, Consulting Group, INC, Suite 210. Minneapolis, Minnesota, 1991.

Gunn M.J., Satkunananthan A., Clayton C.R.I. - Finite element modelling of installation effects. Proceedings of the conference Retaining Structures, organized by the Institution of Civil Engineers and Held at Robinson College,
Cambridge, 20-23 July 1992, pp. 46-55.

Higqins K.G., Potts D.M. Symons I.F. Comparison of predicted and measured performance of the retaining walls of the Bell Common Tunnel, Transport and Road Research Laboratory, Department of Transport, Report 124, 1989.

Ng C.W.W., Lings M.L., Simpson B., Nash D.F.T. - An approximate analysis of the three-dimensionnal effects of diaphragm wall installation. Geotechnique, 1995, 45, no 3, pp. 497-507.

Potts D.M., Fourie A.B. - The behaviour of a propped retaining wall : results of a numerical investigation. Geotechnique, 1984, 34, n 3, pp. 383-404.

Powrie W. Kantartzi C. - Installation effects of diaphragm walls in clay. Proceedings of the conference Retaining Structures. organized by the Institution of Clvil Engineers and Held at Robinson College, Cambridge, 20-23 July 1992, pp. 37-45.

Powrie W, Li E.S.F. - Finite element analyses of an in situ wall propped at formation level. Geotechnique, 1991, 41, $n^{\circ}$ 4. pp. 499-514. 